

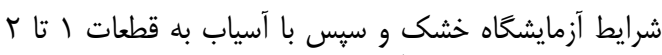

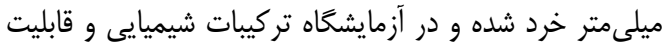

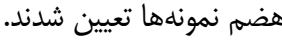

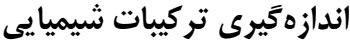

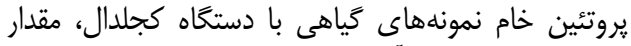

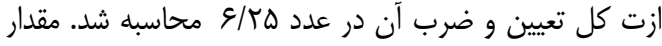

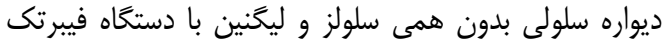

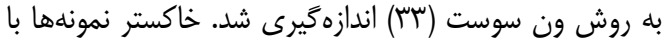

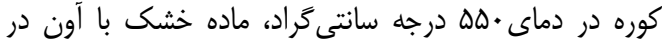
دماى ه • ا درجه و هربى خام با دستخاه سوكسله تعيين شد.

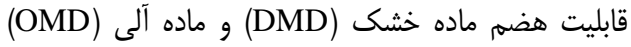

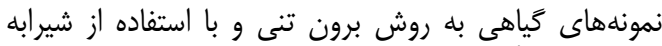

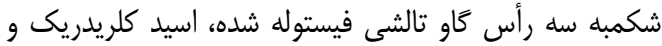

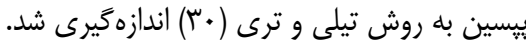

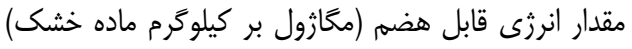

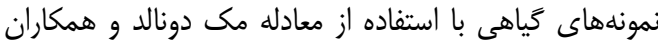

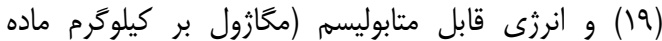

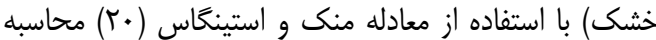

$\mathrm{DE} \mathrm{MJ} / \mathrm{Kg}=0.019(\mathrm{DOMD}) \times 10$ $\mathrm{ME} \mathrm{MJ} / \mathrm{Kg}=0.0157(\mathrm{DOMD}) \times 10$

آناليز آمارى براى انجام اين يزوهش إز إز روش تجزيه وارئ إيانس استفاده

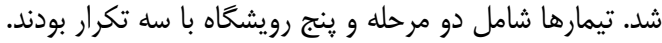

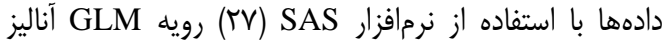

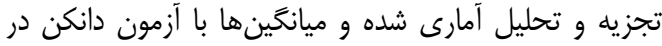
سطح خطاى ه درصد مقايسه شدند.

\section{نتايج و بحث}

تجزيه واريانس تركيبات شيميايى علوفه بارهنى ياكلاغى

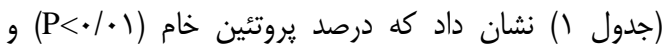

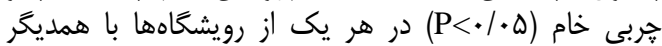

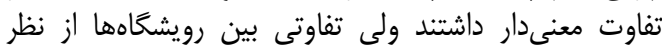
درصد ماده خشك، ديواره سلولى بلدون همى سلى سلولز و ليخنين رونين

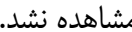

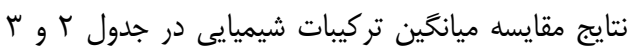

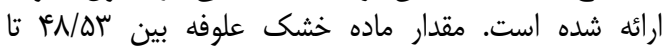
•

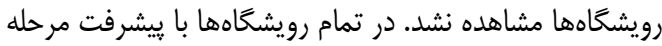

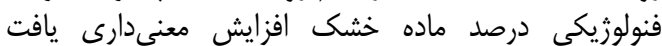

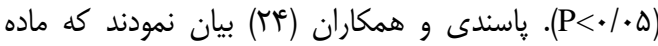

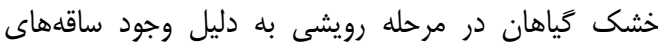

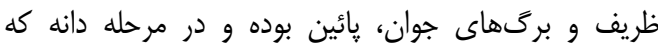

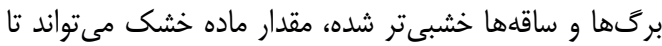

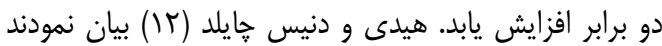

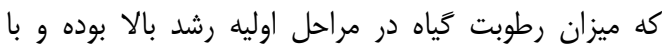

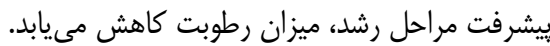

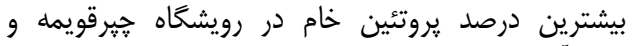

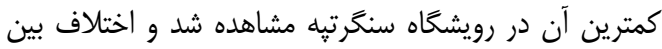

\section{كَياهشناسى و مناطق يراكنش}

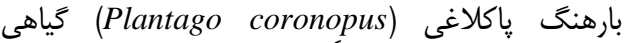

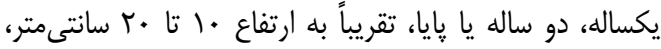

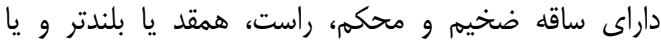

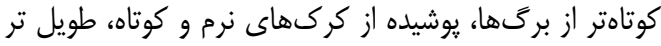

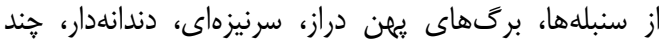

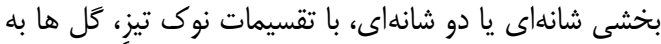

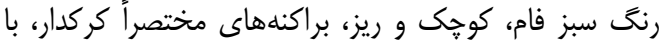

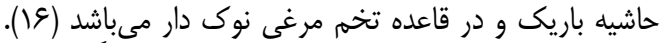

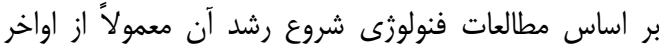

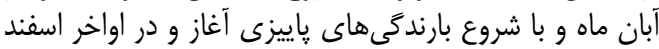

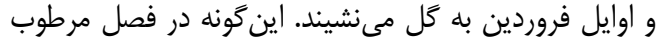

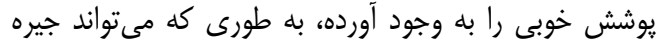

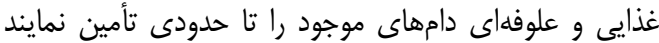

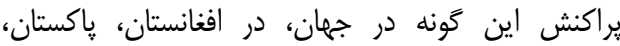

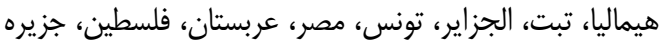

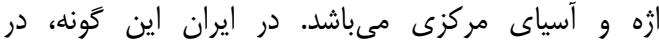

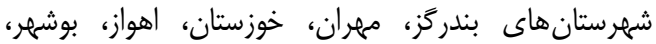
كازرون و سيستان زيراكنش دارد (ع) (1).

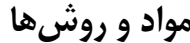

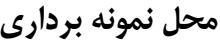
منطقه مورد مطالعه در شمال شرد شرق استان كلستان قرار

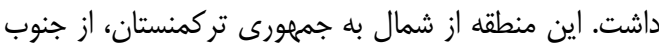

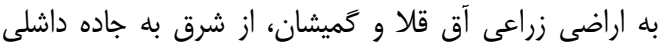

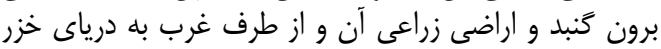

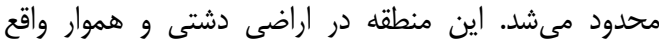

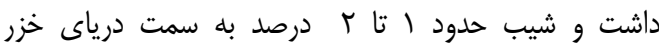

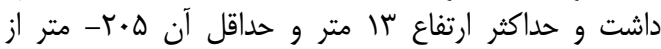

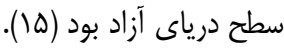

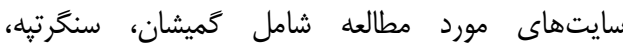

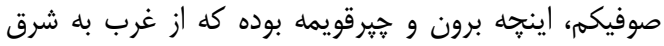

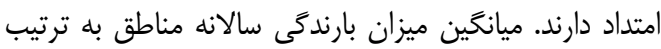

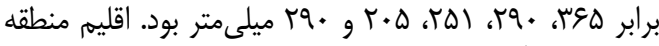

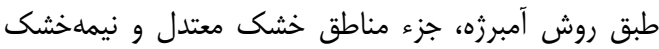

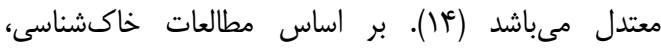

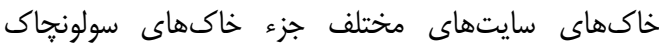

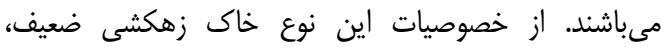

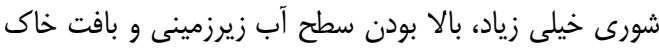

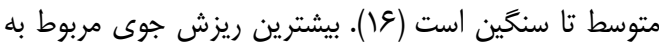

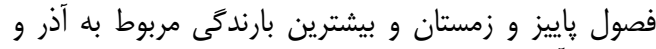

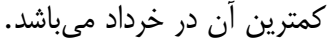

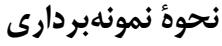

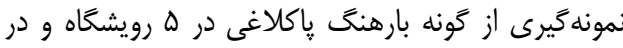

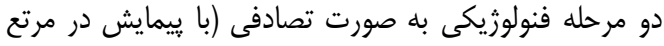

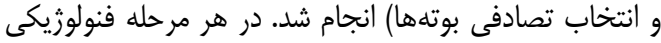

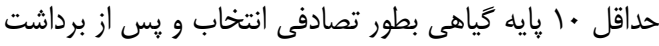

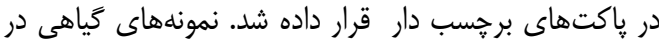


بيشرفت مراحل رشد، مقدار آن افزايش يافت كه در اين زمينه

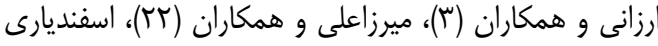

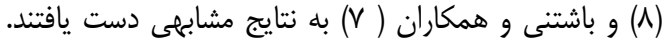

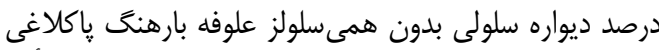

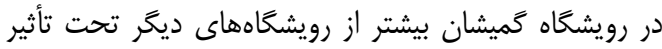

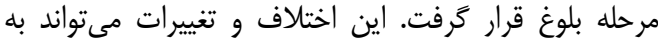

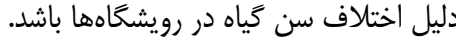

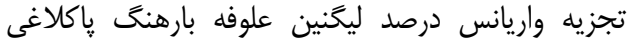

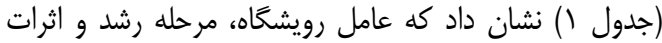

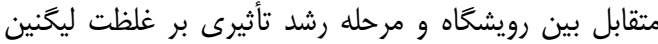

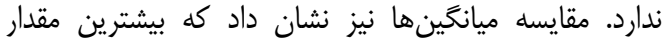

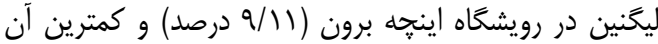

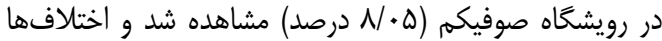

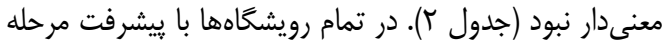

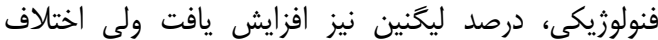

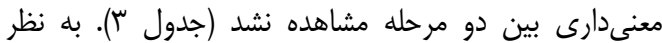

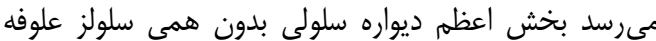

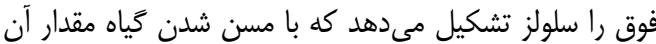

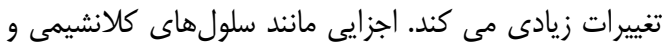

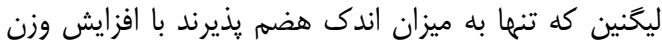

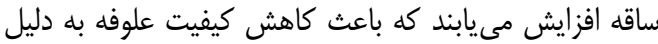

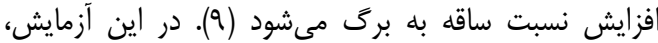

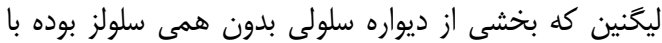

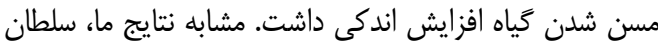

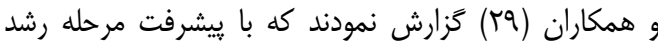
درصد ليكنين افزايش مي ميابد.

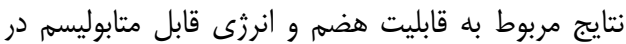

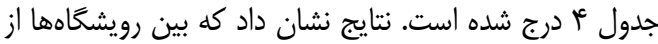

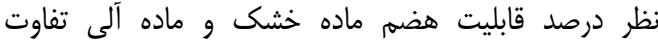

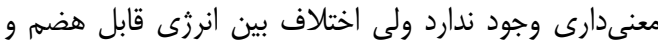

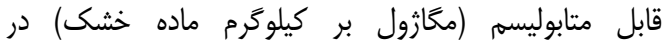

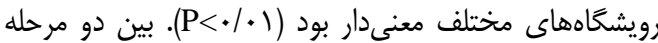

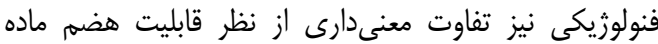

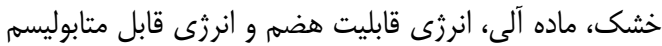

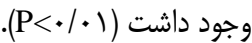

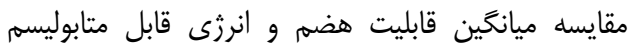

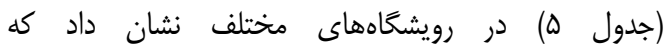

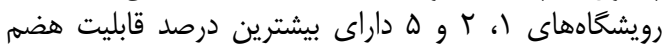
ماده خشك ( به ترتيب أو

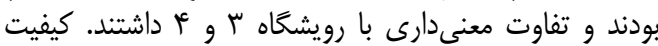

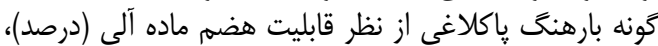

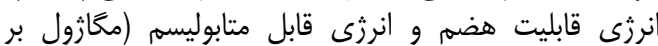

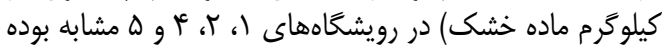

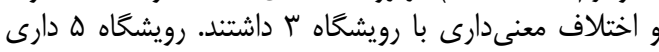

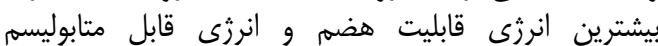
(مكازول بر كيلوَّرم ماده خشك) بود.

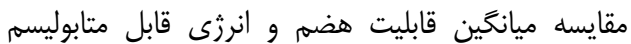

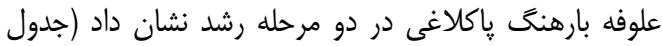
ع) كه قابليت هضم علوفه در همه رويشكاهها با بيشرفت رئان

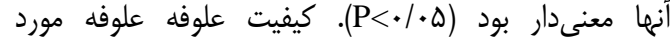

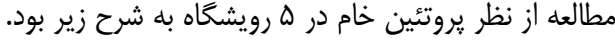

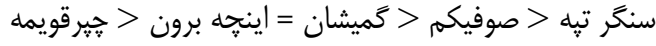

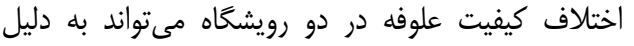

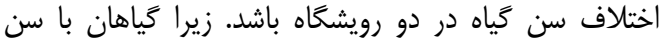

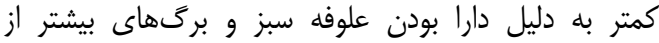

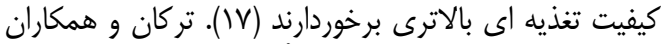

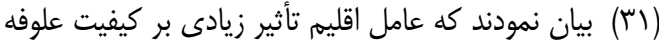

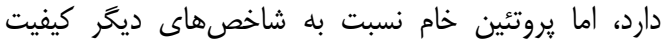

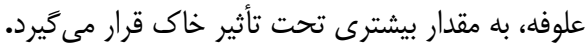

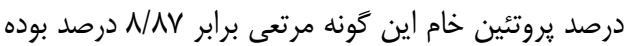

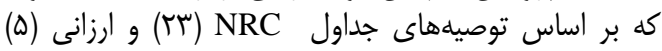

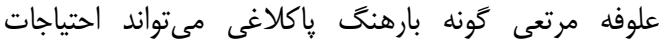

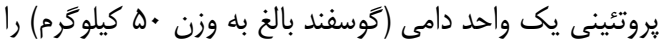

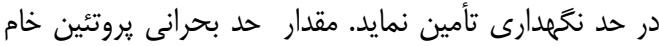

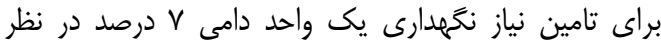

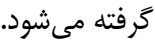

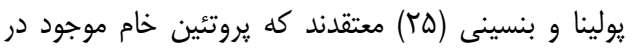

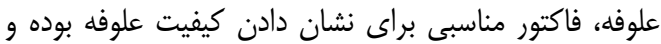

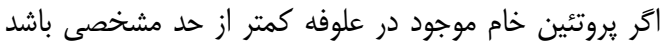

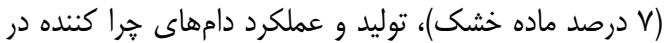
مرتع كاهش مى ميابد.

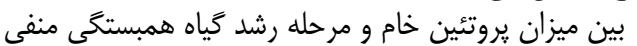

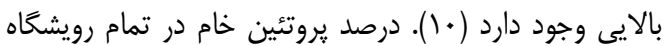

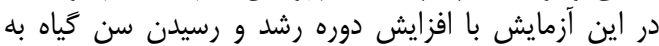

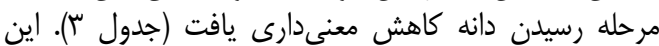

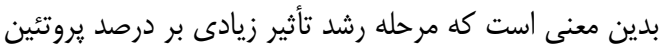

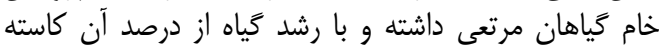

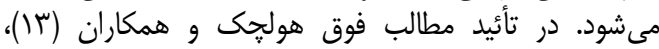

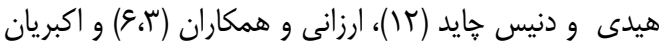

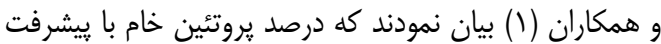

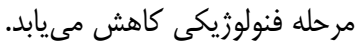

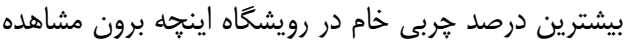

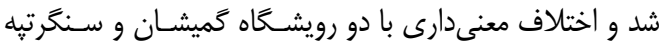

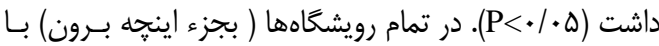

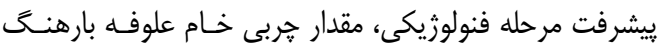

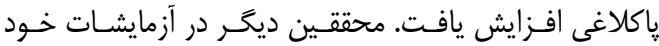

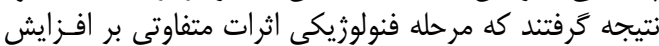

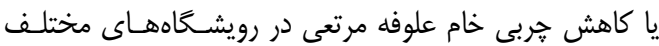

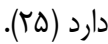

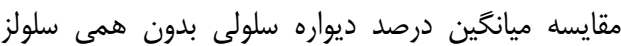

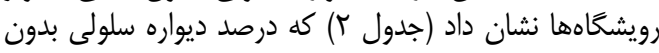

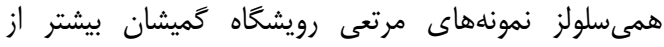

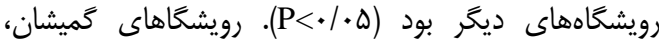

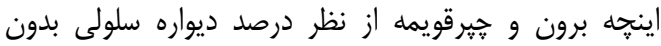
همى سلولز مشابه همديكر بودند.

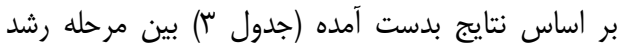

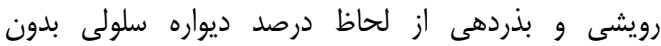

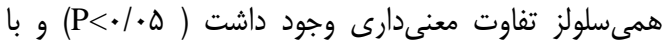


كيفيت علوفه در ه رويشگاه از نظر درصد يروتئين خام، درصد

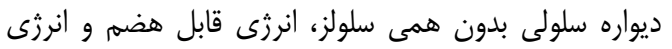

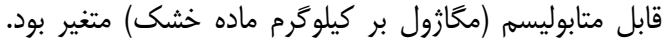

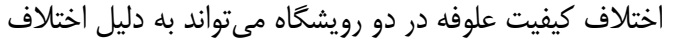

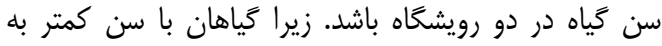

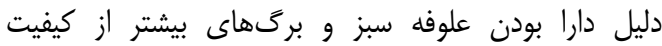

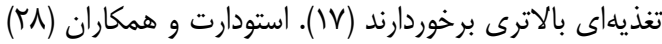

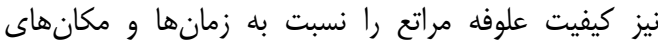

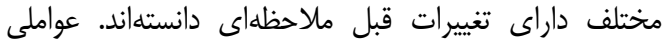

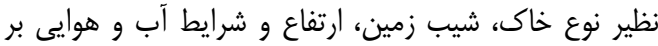

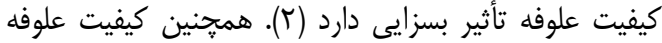

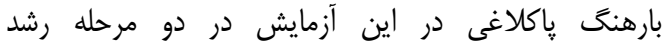

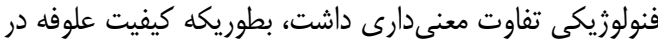

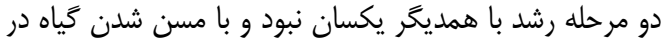

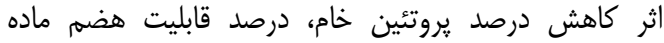

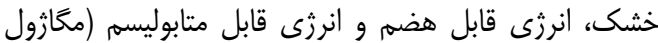

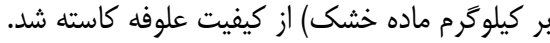

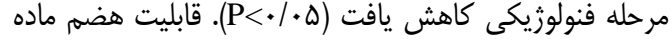

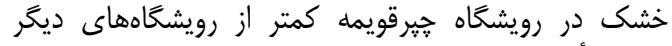
تحت تأثير مرحله رشد قرار كرفت. همانند قابليت هضماهم،

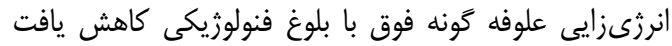

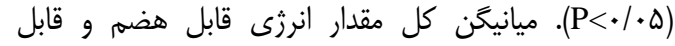

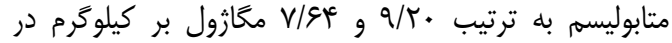

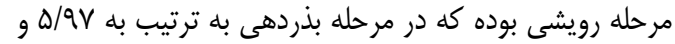

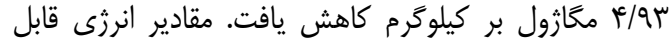

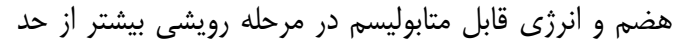

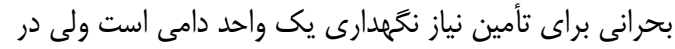

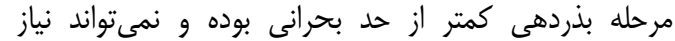

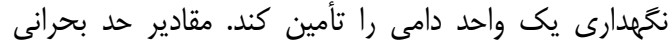

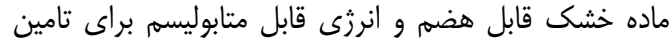

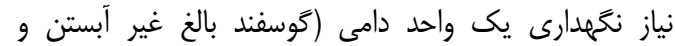

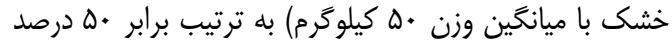

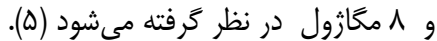

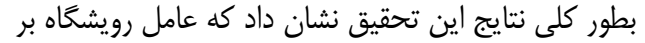

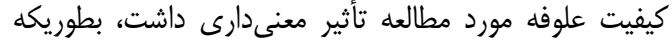

جدول ا- نتايج تجزيه واريانس تركيبات شيميايى علوفه يلانتاكو Table 1. Analysis of variance of chemical composition of Plantago Coronapos

\begin{tabular}{|c|c|c|c|c|c|c|c|}
\hline \multirow[b]{2}{*}{ ADL } & \multicolumn{4}{|c|}{ ميانگين مربعات } & \multicolumn{3}{|c|}{ درجه } \\
\hline & $\mathrm{ADF}$ & $\mathrm{OM}$ & $\mathrm{EE}$ & $\mathrm{CP}$ & DM & آزادى & منبع تغيير \\
\hline $1 / .9$ & $\Gamma \Delta / \widetilde{r V}$ & $T / / Q$ &.$/ 99$ & $\Gamma / \Delta \omega$ & $V / \Delta S$ & f & رويشگاه \\
\hline$g / V \pi$ & $\| \Lambda \Delta / V$. & rIV/GY & .1 .9 & שואוזו & rATIV/. I & 1 & مرحله رشد \\
\hline$r / 9 F$ & $9 Y / / F$ & 1N/9D & $\cdot / f F$ & $\cdot / T \Lambda$ & $q / 4 \Delta$ & f & رويشكاه X كونه \\
\hline$r / T e$ & IN/DT & س & $1 / \pi r$ & $\cdot / V T$ & $I r / \Delta F$ & $r$. & خطا \\
\hline \multicolumn{8}{|c|}{ محاسباتى f } \\
\hline . & $1 / 91^{\mathrm{ns}}$ & $1 / 4 \Lambda^{\mathrm{ns}}$ & $r / . r$ & $f / 91^{* *}$ & $\cdot / \Delta s^{\mathrm{ns}}$ & f & رويشكاه \\
\hline$r / \cdot \Lambda^{\mathrm{ns}}$ & $94 / \cdot r^{* *}$ & $1 \omega / \Lambda \Lambda^{* *}$ & $\cdot / r \Lambda^{\mathrm{ns}}$ & $1 \Lambda \cdot / 9 \Lambda^{* *}$ & $r \cdot \Lambda r / A r^{* * *}$ & 1 & مرحله رشد \\
\hline.$/ 9)^{\mathrm{ns}}$ & $r / q \Lambda^{n *}$ & $I / T^{\mathrm{ns}}$ &.$|8|^{\mathrm{ns}}$ & $\cdot / / q^{n s}$ & $\cdot / \mathrm{v}^{\mathrm{ns}}$ & f & رويشكاه × كَونه \\
\hline-- & -- & -- & -- & -- & & r. & خطا \\
\hline
\end{tabular}


جدول r- مقايسه ميانخين تر كيبات شيميايي علوفه بارهنَ پاكلاغى در ه رويشخاه Table 2. Comparison of chemical composition of Plantago Coronapos in five habitats

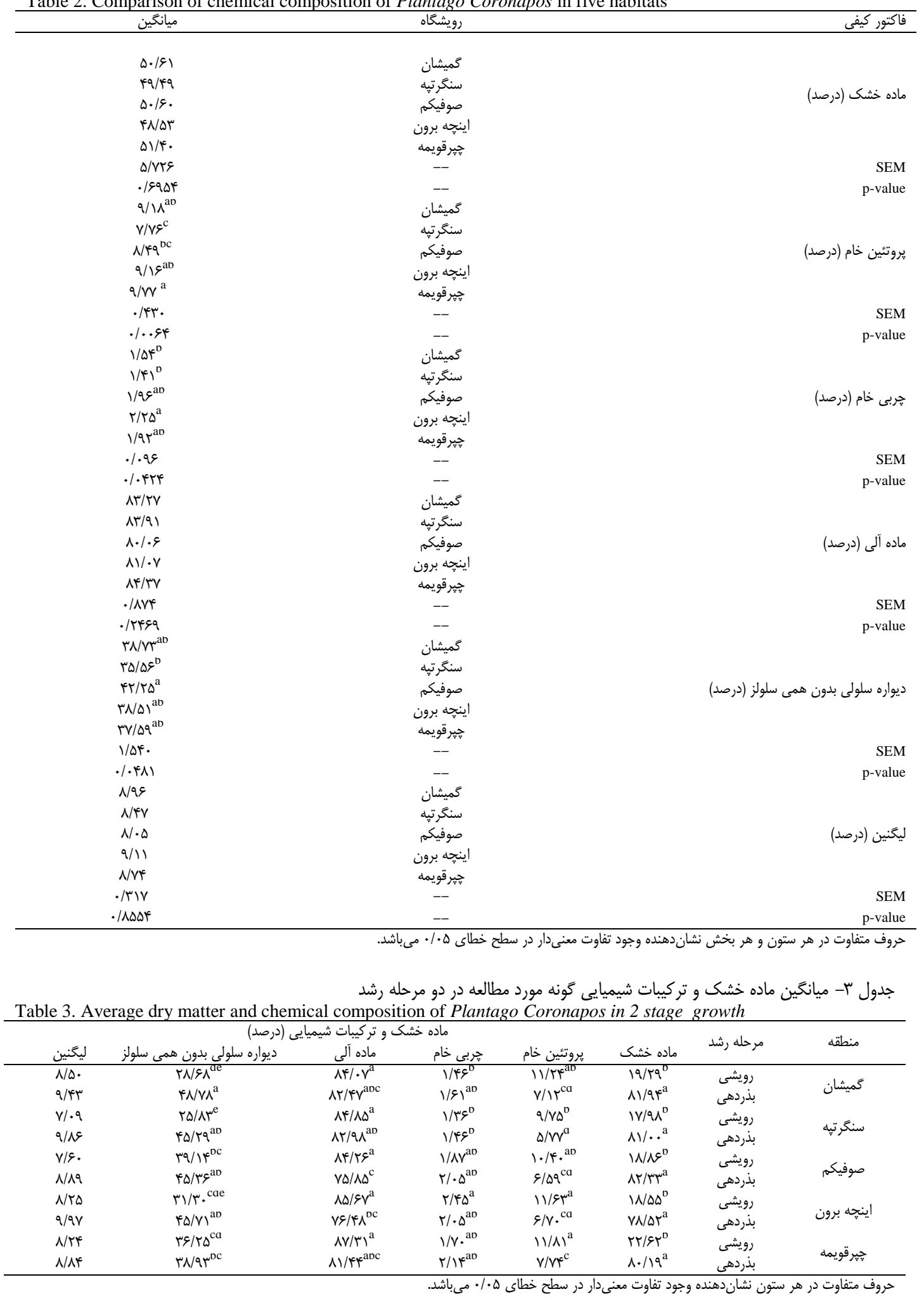


جدول عأ- نتايج تجزيه واريانس قابليت هضم و انرزى قابل متابوليسم علوفه بارهنَ ٍِاكلاغى Table 4. Analysis of variance of digestibility and metabolism energy of Plantago Coronapos

\begin{tabular}{|c|c|c|c|c|c|c|c|c|c|}
\hline \multirow[b]{2}{*}{ ME } & \multicolumn{2}{|l|}{$\mathrm{F}$} & \multirow[b]{2}{*}{ DMD } & \multirow[b]{2}{*}{ ME } & \multicolumn{2}{|c|}{ ميانكَين مربعات } & \multirow[b]{2}{*}{ DMD } & \multirow{2}{*}{ آزادى } & \multirow[b]{2}{*}{ منبع تغيير } \\
\hline & $\mathrm{DE}$ & OMD & & & $\mathrm{DE}$ & OMD & & & \\
\hline & $\kappa / \lambda f^{* \omega s}$ & $r / 9 q^{\text {ns }}$ & $r / / T^{\text {ns }}$ & & & & & & \\
\hline$f / \wedge f^{* * *}$ & & & & $r / q q$ & $\Delta / \mp 1$ & $19 \cdot / F \Delta$ & $9 \Delta / 4 \varphi^{2}$ & f & رويشگاه \\
\hline$V \cdot / 10^{m m}$ & $V \cdot / T^{N T}$ & $f \cdot / \mu f^{c *}$ & $r N / \mu \omega^{n T}$ & & & & & & \\
\hline \multirow{4}{*}{$r / \mu Y^{n s}$} & & & & $\Delta T / \Delta V$ & $V N / F V$ & $M F \cdot r / \Delta T$ & $r \mid g V / r \mu r$ & 1 & مرحله رشد \\
\hline & $r /$ rrns & $1 / 99^{* \pi}$ & $r / .^{\text {ns }}$ & & & & & & \\
\hline & & & & IN/VG & $r / \Delta Q$ & $\| \varepsilon / M$ & 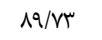 & f & رويشگاهXمرحله رشد \\
\hline & & & & $\cdot /$ V & $1 / 1 r$ & $\Delta Q / \Delta \Delta$ & $\kappa \mu / \Lambda \mu$ & r. & خطا \\
\hline
\end{tabular}

جدول ه- مقايسه قابليت هضم و ارزش انرزىزايى علوفه بارهنگ پاكلاغى در ه رويشگاه Table 5. Comparison of digestibility and metabolism energy of Plantago Coronapos in five habitats

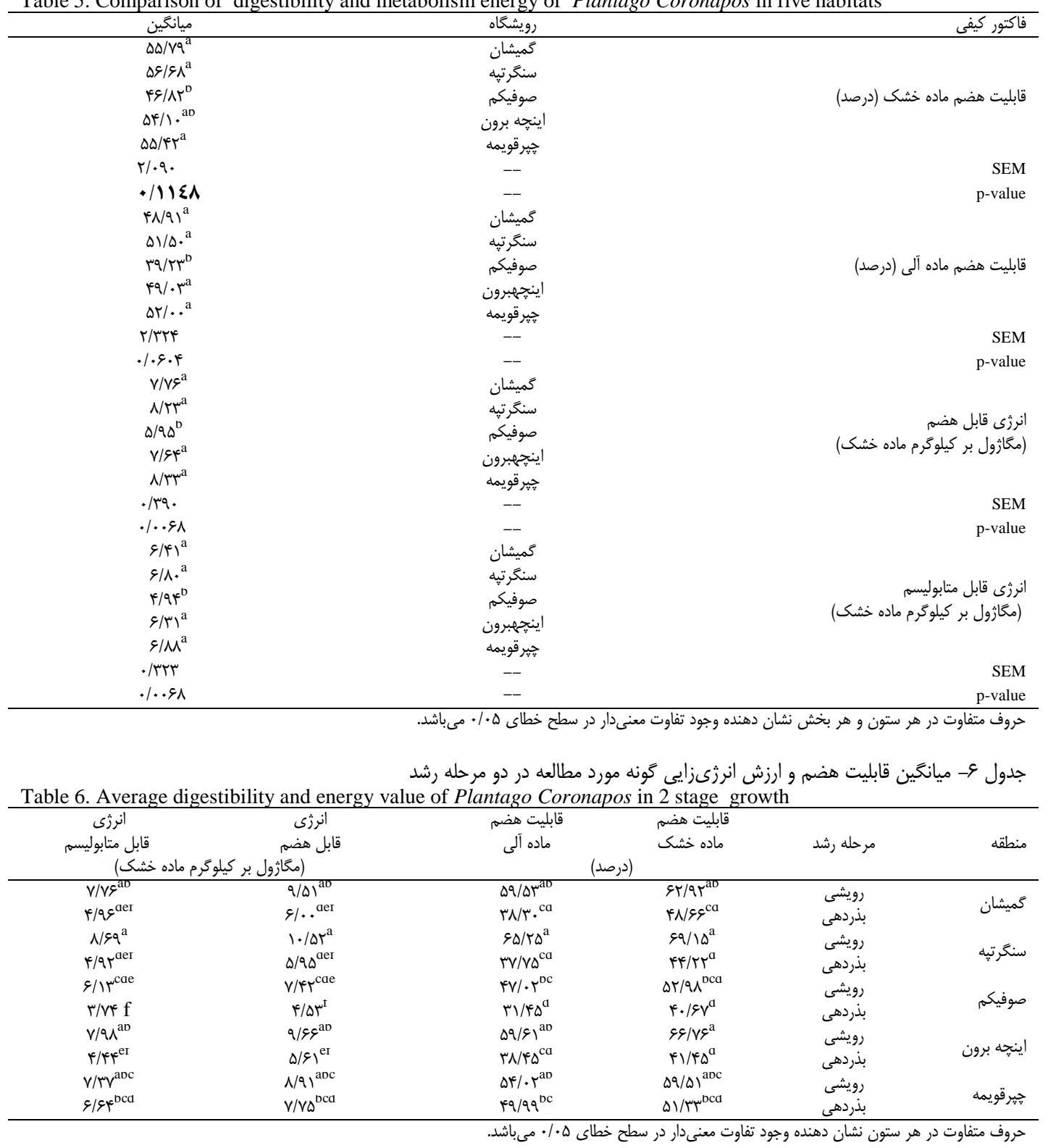


1. Akbarian $\mathrm{H}$ and M. Yosefali. 2015. Determination of Salsola vermicolata and Suaeda fruticosa forage quality of sistan region at different Phenological Stage.Research and Animal Production, 11: 92-101.

2. Arzani, H., J. Torkan, M. Jafari, A. Jalili and A.Nikkhah. 2001. Effects of phenological stages and ecological factors on forage quality of some range species. Iranian Journal of Agricultural Science, 32: 385399.

3. Arzani, H., M. Mosayebi and A. Nikkhah. 2009. Determination of animal unit size and animal unit requirement of fashandy sheep breed grazing on rangelands (Case Study: Taleghan). JWSS - Isfahan University of Technology, 12: 349-360.

4. Arzani, H., H. Piri Sahragard, J. Torkan and K. Saedi. 2010. Comparison of fenological stages on forage quality of rangelands species in rangeland of Saral Kordestan, Iranian Journal of Rangeland, 4: 160-167.

5. Arzani, H., J. Motamedi and M.A. Zare Chahoki. 2010. Report of national project "Forage quality of range species in Iran", Organization of Forests, Rangelands and Watershed Management of Iran, $230 \mathrm{pp}$.

6. Arzani, H., H. Pouzesh, J. Motamedi, R. Mirakhorli and S.A. Niknejad. 2012. Effects of phenological stages on forage quality of five rangeland species in semi-steppe rangeland of Jashlobar Semnan. Iranian Journal of Range and Desert Research, 3: 384-394.

7 . Bashtani, M., J. Farzadmehr, O. Ghafori, N. Afzali and M. Sharifi. 2015. Effect of growth stage and processing by $\mathrm{NaOH}$ and $\mathrm{Ca}(\mathrm{OH}) 2$ on chemical composition and degradation parameters of Haloxylon $s p$. Pasture plant in seeding stage. Research and Animal Production, 12: 96-104.

8. Esfandyari, A. 2005. Determination of animal unit equivalent (AUE) and daily requirement for Sanjabi sheep breed (Case Study: Kermanshah).M.Sc. Thesis, Department of Natural Resources, Tehran University, $85 \mathrm{pp}$.

9. Fahey, J.R.C. 1994. Forage quality, evaluation and utilization, American society of Agronomy, $998 \mathrm{pp.}$

10. George, M.R. and M.E. Bell. 2001. Using stage of maturity to predict the quality ofannual range forage. ANR publication 8019. Rangeland management series. Davis,CA:University of California, 7 pp.

11. Harris, Lorin E. 1950. The nutritive value of range forage as affected by vegetation type, site, and state of maturity. Utah Agric Expt Sta Bull, 45 pp.

12. Heady, H.F. and R. Dennis Child. 1994. Rangeland ecology and management. West View Press, 520 pp.

13. Holchek, J.I., C.H. Herbal and R.D. Pieper. 2004. Range management principles and practices. Prentice Hall Pub. USA. Forth Edition, $587 \mathrm{pp}$.

14. Hosseini, S. 2005. Autecology of Halocnemum strobilaceumin Golestan Province. Final report of research plan, natural resources research centre of Golestan Province, $50 \mathrm{pp}$ (In Persian).

15. Hosseini, S., A.A. Shahmoradi and G. Abarsaji. 2007. An Investigation on the presence form of halocnemum strobilaceum in saline and alkaline rangelands of northern Golestan province. Iranian journal of Range and Desert Reseach, 14: 110-123.

16. Hosseini, S.A. and M. Tavan. 2010. Saline and alkali plants of Golestan rangelands. University Jahad Publishing Center, Tehran, $156 \mathrm{pp}$.

17. Kermit, O. 1956. Factors affecting the nutritive value of range forage. Journal of Range Management, 6: 220-224.

18. Launchbaugh, K.L., J.W. Stuth and J.W. Holloway. 1990. Influence of range site on diet selection and nutrient intake of cattle. J Range Manage, 43: 109-166.

19. McDonald, P., R.A. Edwards, J.F.D. Greenhalgh and C.A. Morgan. 1995. Animal Nutrition. (5 ${ }^{\text {th }}$ ed.), Longman Group Ltd: London, UK., pp: 266-283.

20. Menke, K.H. and H. Steingass. 1988. Estimation of the energetic feed value obtained from chemical analysis and gas production using rumen fluid, Animal Research Development, 28: 7-55.

21. Minson, D.J. 1987. Estimation of the nutritive value of forage, in temperate pastures, their production, use and management. Eds. J.L. Wheeler, C.J. Pearson and G.E. Roberts, Australian Wool Corporation, 415422.

22. Mirzaali, A.T., E. Mirzaali and M.R. Frozeh. 2008. Study of effects of phonological stages on forage quality of two halophyte species of Halocnemum strobilaceum and Halostachys caspica in Gomishan ranges, Pajouhesh \& Sazandegi, 78: 79-84.

23. National Research Council. 1985. Nutritional requirements of domestic animals. Nutrient requirements of sheep ( $6^{\text {th }}$ ed.) Nat. Acad. Sci., National Research Council, Wash., D.C.

24. Pasandi, M., A. Kavian and M.A. Dorri. 2010. The nutritive value of Lathyrus sativus forage cut at four stages of growth in Gorgan. Animal Sciences Journal (Pajouhesh and Sazandegi), 89: 28-32.

25. Pulina, G. and R. Bencini. 2004. Dairy sheep nutrition, CABI publishing, London, 222 pp.

26. Rasouli, B., B. Amiri, M.H. Assareh and M. Jafari. 2011. Determination of nutritive value of Seidlitzia rosmarinusat different phonological stages in three different sites, Iranian journal of Range and Desert Reseach, 18: 32-41.

27. SAS Institute. 1997. SAS user s guide, version 7. SAS Inst., Ine. Cary, North Carolina, USA

28. Stoddert, L.A., A.D. Smith and T.W. Box. 1975. Range management, $3^{\text {rd }}$ edn, Mcgraw-Hill Company New York, $532 \mathrm{pp}$.

29. Sultan, J.I., I.U. Rahim, H. Nawazand M. Yaqoob. 2007. Nutritive value of marginal land grasses of Northern Grasslands of Pakistan. Pak. J. Bot, 39: 1071-1082.

30. Tilley, J. and R. Terry. 1963. A two stage technique for the in vitro digestion of forages crops, J. BrGrasslSoc, 18: 104-111.

31. Torkan, J. and H. Arzani. 2009. Effect of soil on forage quality in different climatic zones. $4^{\text {th }}$ National conference on pasture, rangeland Iran, 112-116.

32. Uniyal, S.K., A. Awasthi and G.S. Rawat. 2005. Biomass availability and forage quality of Eurotia ceratoides Mey in the rangelands of Changthang, eastern Ladakh, Current Science, 89: 201-205.

33. Van Soest, P.J. 1994. Nutritional Ecology of the Ruminant, $2^{\text {nd }}$ ed. Cornell University Press, Ithaca, N.Y, $476 \mathrm{pp}$. 


\title{
Investigation of Plantago Coronopus Forage Quality at Different Phenological Growth Stages in Five Habitats of Golestan Province
}

\author{
Mokhtar Mohajer ${ }^{1}$, Abdolah Kavian ${ }^{2}$ and Mohammad Pasandi ${ }^{3}$ \\ 1 and 2- Assistant Professor and Research Instructor, Agriculture and Natural Resources Research Center, Golestan \\ Province, Agricultural Research, Education and Promotion Organization, Gorgan, Iran \\ 3- Research Instructor of Agriculture and Natural Resources Research Center, Golestan Province, Agricultural \\ Research, Education and Promotion Organization, Gorgan, Iran (Corresponding author: mhm_pasandi@ yahoo.com) \\ Received: October 25, 2015 \\ Accepted: January 30, $201 \overline{6}$
}

\begin{abstract}
Range species forages have different quality and feeding value in different site and times. Therefore research carried out to Investigate of Plantago Coronopus Forage Quality at different phenological Growth Stages in Five Habitats of Golestan Province (Goomishan, soofikam, Sangar Tappeh, Inchehbroon and chaparghoymeh). Samples were collected from two phonological stages (vegetative and seed ripening) and then crude protein (CP), ether extraction $(\mathrm{EE})$, organic mather (OM), acid detergent fiber (ADF) and lignin (ADL) were determined. Dry matter digestibility (DDM) and organic matter digestibility (DOM) of forages were determined using rumen fluid from buffalo by in vitro techniques. The results of chemical analysis indicated that $\mathrm{CP}, \mathrm{EE}$ and $\mathrm{ADF}$ percent were significant differences in different sites $(\mathrm{p}<0.05)$. CP $(\%)$, DOD (\%), DE and ME (mj/kg) in Chaparghoymeh was better than other site. In all sites, quality of $P$. coronopus were varied at two phonological stage and seed ripening $(\mathrm{p}<0.05)$. DM and ADF content were increased with phonological stages increasing, but CP, DDM, DOM, DE and ME of forage were decreased $(p<0.05)$. CP percent at two growth stages are higher than its critical level (7\%) for one animal unit maintenance. DE and ME amounts at vegetative growth stages are higher and at seeding stage are lower than its approximate amount of critical level for supply daily requirement of animal unit. In general, result shown that forage quality of rangelands were varied in different sites and this plant at vegetative stage can supply protein and a great part of energy requirement of different grazing ruminant groups (sheep and goat).
\end{abstract}

Keywords: Golestan Province, Nutritive value, Phonological stage, Plantago coronopus 